\title{
M.T.S.: An adaptable microcomputer-based testing system
}

\author{
DON D. FOREE, DAVID A. ECKERMAN, and SHARON L. ELLIOTT \\ University of North Carolina, Chapel Hill, North Carolina
}

\begin{abstract}
An Apple II+ computer-based human testing system is described. The system, which was developed using the Pascal language, provides library units of low-level procedures to rapidly access a clock, collect responses, control video output, control a touch-sensitive monitor, and display high-resolution graphics. Built-in hardware and software checks provide a high degree of system reliability. Use of easily altered text files for control of trials within a task and inclusion of and ordering of tasks within a session allows the system to be readily adapted.
\end{abstract}

The M.T.S. system is an Apple IIt computer-based testing system developed to administer and collect data from a variety of behavioral tasks using human subjects. The system was developed in cooperation with the Environmental Protection Agency to be used as a tool to assess toxic effects of environmental pollutants, but is applicable to a wide variety of research and teaching situations encountered in psychology. M.T.S. is written in Apple Pascal (version 1.1), with some time-critical portions written in 6502 assembly code. M.T.S. makes use of many of the features of Apple Pascal, such as program segmentation and procedure libraries, to produce a cohesive testing system that is easily adaptable and expandable. Each task is a separate module that may be easily included or not in a particular testing situation. The system provides hardware and software checks to ensure that all tasks included in a particular testing session will run to completion. Extensive use of the Apple HIRES graphics mode is employed to provide a wide latitude in choice of and a fine control in presentation of visual stimuli. The M.T.S. system also provides ancillary programs to facilitate data storage and data transfer for analysis on larger computer systems.

\section{HARDWARE AND SOFTWARE REQUIREMENTS}

Minimum hardware requirements for the M.T.S. system are an Apple II or equivalent (e.g., Franklin Ace 1000) computer with $64 \mathrm{~K}$ of random access memory (RAM) (note: for the Apple II and Apple II+ computers, this implies the use of a 16K RAM card or

This project was supported through Cooperative Agreement CR808834020 with the Environmental Protection Agency. Apple and Apple II are trademarks of Apple Computer, Inc. Franklin Ace 1000 is a trademark of Franklin Computer Corporation. Pascal is a trademark of the Regents of the University of California at San Diego. The M.T.S. system and all of its library units, ancillary programs and individual procedures are copyrighted by Don Foree. Requests for reprints should be directed to Don Foree, Psychology Department, University of North Carolina, Chapel Hill, NC 27514. "language" card installed in backplane slot 0), a Mountain Computer Company Apple Clock, two 5.25-in. floppydisk drives, a color monitor, and a set of headphones.

In addition, three input manipulanda consisting of a box with two push buttons and a separate foot switch are needed, and some minor hardware modifications must be made to the computer. The modifications include externalizing the game input/output port for ease of adding and removing devices that are accessed through this port, externalizing the auditory output and adding a volume-controlled headphone jack, and modifying the video circuitry to allow greater control over timing of video events (Reed, 1979). These modifications are simple and reversible and require no knowledge of electronics. They are described in M.T.S. Technical Notes (Technotes) 1, 2, and 3 in a manner that should allow any person with simple soldering skills to complete them. (The technical notes are available on request; see the section on availability.)

This minimum hardware configuration allows use of the system for tasks involving only push-button input and a few rapid-access graphics displays. Extensive and elaborate tests may be generated with this minimum system; however, to take full advantage of the capabilities of the M.T.S. system, additional hardware should be included. Many of the tasks that have been implemented require at least some of these additional items. The data and program-storage capabilities provided by two 5.25-in. floppy-disk drives are minimal. Optimally, the system should have 2 megabytes of mass storage on line (e.g., a pair of double-sided, double-density 8-in. floppy-disk drives). Another alternative is to use three or possibly four 5.25-in. floppy-disk drives. Because of the large volume of data that may be collected, the use of fixed-medium mass-storage devices is not recommended unless backup is easy and readily available. In order to obtain full benefit of the system's graphics capabilities, a 128K RAM card should be employed. (The M.T.S. software supports RAM cards from Legend Industries Ltd., Pontiac, MI, and Prometheus Products, Fremont, CA, or any other RAM cards that utilize the same mem- 
ory bank-switching scheme.) Note that the cost of this card is partially offset, since it may replace the $16 \mathrm{~K}$ RAM card in slot 0 of the backplane of the computer. Several of the tasks currently implemented make use of a special touch-sensitive color monitor as an additional response manipulandum. (The touchscreen that is supported is marketed by the International Institute of Applied Technology, 20010 Century Boulevard, Suite 100, Germantown, MD 20874.) As in the case of the $128 \mathrm{~K}$ RAM card, the cost of this item is partially offset, since it replaces the color monitor in the system described above.

A third class of hardware options is suggested for data analysis or development of additional tasks for the system. These include an 80-column communicationsinterface card that allows viewing of two monitors simultaneously (one for text and one for graphics) and a monochrome monitor, a card for serial transfer of data via the RS-232 protocol, a printer, and a modem.

In order to install and run the M.T.S. software, it is necessary to have the Apple Pascal System (version 1.1).

\section{SYSTEM OVERVIEW}

The main M.T.S. program is a relatively short segment of code that defines global data structures, global variables, and a few procedures that are common to most of the individual tasks. The primary purpose of the main M.T.S. program is to control sequencing of the presentation of individual tasks.

Each task is a SEGMENT PROCEDURE within the main program. Thus, the code for each task resides on disk until that particular task is called for, at which time it is brought into computer memory. This segmentation allows the M.T.S. program to be many times larger than the capacity of the computer's memory.

Low-level procedures and functions that are used frequently by the program have been grouped into logical units and installed in the SYSTEM.LIBRARY. These INTRINSIC UNITS may be considered as an extension of the Pascal language and may simply be called by name for use. By exercising options at the time that the M.T.S. program is compiled, the user may control which units are in computer memory at any particular point in the execution of the program. As with the task segments, unused units reside on disk instead of requiring computer memory. At this time, three units have been developed. The first, called MTSSTUFF, includes procedures that read input from response manipulanda, procedures that quickly read and format information from the clock, and a procedure that presents auditory stimuli. The second unit, MTSGRAFIX, contains procedures that control the HIRES graphics screen. Finally, the TOUCHRAM unit contains procedures that gather input from the touchscreen and that quickly access pictures stored on the 128K RAM card. These units are completely com. patible with other units (e.g., TURTLEGRAPHICS and
APPLESTUFF) in the SYSTEM.LIBRARY that is provided with the Apple Pascal system. The procedures and functions included in these units are described briefly in Appendix A.

Separate from the main M.T.S. program are a group of utility programs that may be used to test components of the system hardware and to convert raw data into text files that may be sent to a printer or transferred to another computer over telephone connection or over a dedicated serial line at high speed. In addition, a series of procedural steps has been developed to analyze data from the various tasks on large mainframe computers via the SAS (Statistical Analysis System) package.

\section{EXPERIMENTER INTERACTION WITH THE SYSTEM}

Interaction of the experimenter with the M.T.S. system may take place at three levels. At the first and simplest level, running a subject is as simple as inserting a system disk and a data disk, turning on the computer, and responding to a few questions that appear on the monitor. An initialization portion of the M.T.S. program initializes the system, checks the hardware, finds all files that will be needed to complete the series of tasks to be administered in the session, and assures that enough room is available on the data disk to accommodate all of the output files from the session. At various points in the initialization process, the experimenter is prompted to interact with the system by pressing buttons, touching the touchscreen, or confirming date and subject identification codes. A subject identification code is updated by the system and assigned to the subject for the session. The identification code consists of a subject number, an experimenter character, and a test series character. This identification code is prefixed to all output files created during a session to produce a unique and informative name for each data file. The system checks to assure that the new file names will not conflict with names of any existing files on the data disk. If any problems occur in the initial system checks, diagnostic messages appear on the monitor to explain the problem. The initialization process is very easy to learn and ensures that none of the many steps necessary for a session to run to completion will be overlooked. This allows people with little or no computer experience to confidently supervise experimental sessions.

At a second level, a person with some knowledge of the Pascal system's editor and filer programs can configure a session or customize a task by changing input files. The flow of a session is controlled by the contents of a simple Pascal text file that contains the names of the tasks to be run in the order in which they are to be run. The number of tasks or order of tasks may be quickly and easily changed by changing the contents of the control file. Similarly, the number of trials in a particular task and the stimuli that will be presented on a particular trial are controlled by the contents of one or 
more input text files associated with that task. Several versions of the same task and sometimes completely different tasks can be created simply by using the Pascal editor to change or create new task input files.

Two features available in the currently implemented tasks are instruction and practice options. The form that these options take may be altered by a user at this second level of interaction. For instance, at the beginning of each session, the subject is presented with a short training segment that explains the use of the foot pedal and push buttons. The subject is then given a very brief training drill on buttonpressing that is checked for correctness and repeated if necessary. This training session emphasizes the need for the subject to be accurate and yet respond quickly to screen information. Likewise, most tasks start with a set of instructions that describe the task and the response requirements for the task. Like the input files for a task, these instructions are held in task instruction text files. A procedure within the M.T.S. system code reads the task instruction files and presents the instructions on the HIRES screen. The instructions are paced by the subject, who presses an indicated button to see the next page of instructions. A mechanism has been included to present examples from the task within the instruction stream. Again, modifications can be made to these instruction text files by use of the Pascal editor.

At the third level, an experimenter with knowledge of the Pascal language can alter existing tasks or create new tasks to be added to the system. Guidelines are provided in the system documentation (see section on availability) that ensure that the new tasks will be fully integrated into the system so that full advantage of the systems features may be employed. Since source code of the individual tasks is provided, the programmer may alter existing tasks or use the source code from old tasks as a model for developing completely new tasks. The time to develop a new task or change an existing one depends, of course, on several factors, including how much the new task differs from existing ones and the experience of the programmer. In our experience, a completely new task may be developed, tested, and integrated into the M.T.S. system in about 1 workweek.

\section{DATA OUTPUT AND MANIPULATION}

A common format for trial data output has been established and incorporated within the M.T.S. system. Each task opens one or more disk output files to which data are saved. To ensure that all relevant information is maintained for each trial of a task, the input information (stimuli and other trial-defining information) is stored along with the trials' response information. This strategy reduces doubt about the stimulus conditions of any given trial. Data from individual trials are transferred to disk frequently during a task. Thus, if power outages or other mechanical or electronic problems arise during a testing session, most of the data collected up to the time of the problem are available.

The common format of output data facilitates data analysis. The M.T.S. system contains a separate program that transforms raw data from a compressed form (Pascal data files) to a form (Pascal text files) that may be directly sent to other systems or to a printer. This program is designed so that all of the files on a disk or only specified files of interest are transferred. Another program transmits text file data at high speed (up to 9600 baud) over a dedicated serial line. Again, with this program, the operator may choose files in a variety of ways. The program also allows selection of some transmission parameters to meet requirements of the device to which the information is to be sent. The receiving machine must, of course, be programmed to conform to a simple transmission protocol.

\section{TASKS IMPLEMENTED}

The tasks that have been implemented on the M.T.S. system are listed in Appendix B. The selection represents an effort to develop brief tasks requiring only about a fifth-grade reading ability and providing a broad screening of cognitive abilities that might be impaired by exposure to a toxicant. The tasks are being evaluated as to reliability and stability of performance for subjects of various ages and levels of functioning.

\section{EXPANDABILITY AND ADAPTABILITY}

The M.T.S. system as implemented utilizes Applespecific procedures for presenting stimuli and obtaining responses. This and differences between the Apple Pascal system and other versions of Pascal would make transfer of the M.T.S. system to other computers a nontrivial undertaking. However, when the equipment for which the system was developed is used, expanding and adapting the system to individual needs is a relatively simple matter. As noted above, many alterations in individual tasks may be made simply by changing a task's input and/or instruction files. In other cases, minor alterations to a task's source code and recompilation of the M.T.S. program may be necessary to provide the required effect. Finally, the coding of entirely new tasks and the incorporation of these tasks into the system may be done by following the guidelines in the M.T.S. documentation for adding new tasks and by using existing tasks' source code as examples. These ways of expanding the system require varying degrees of knowledge of the Apple Pascal system and the Apple Pascal language.

Some parts of the system may also be adapted for use in situations other than the ones for which the system was developed. For instance, the initialization portion of the M.T.S. that performs hardware and software checks could be adapted as a user interface to 
animal experiments. To do so, one would need to alter the initialization section to perform any hardware checks that are not presently provided and alter a text file that is a list of files that will be required by the session. The program could then perform many of the tedious but necessary steps, such as file naming, subjectnumber updating, and checking for disk space requirements.

\section{COMPARISON WITH OTHER TESTING SYSTEMS}

There is emerging a rich resource of hardware/ software systems designed to aid in the development of computer programs to carry out psychometric and cognitive testing. This journal has been and will continue to be a source of descriptions of such systems, as will newsletters such as MicroPsych (Department of Psychology, Northern Kentucky University, Highland Heights, KY 41076), Computers in Psychology and Psychiatry (26 Trumbull Street, New Haven, CT 06511), and several others. As a start in helping the potential user to determine which of these systems would best meet his/her particular needs, we will include a brief comparison of M.T.S. with two other systems that are based on the Apple II computer and the Apple Pascal language: the Cognitive Testing Station, marketed by the Digitry Company, Inc., Box 266 River Road, Edgecomb, Maine 04556, and the Apple Pascal Tester, offered by Steven Poltrock and Gregory S. Foltz, Department of Psychology, University of Denver, Denver, Colorado 80208.

Poltrock and Foltz (1982a) introduced an Apple Pascal-based system for conducting individualized psychometric and cognitive testing both in the laboratory and in the field. The system requires an Apple II with some hardware modification (Reed, 1979), a 16K RAM card, the Pascal language, at least one 5.25-in. disk drive, and a Mountain Computer Company Clock with millisecond capability. For cost of materials, they offer a software system, with which one can construct, modify, conduct, and score tests or experiments. The source code for their programs is also included so that a sophisticated user can revise and extend their system.

An interactive program that is part of the system prompts the user in the construction of an input file that will control a single experiment. In constructing the experiment file, the experimenter specifies possible stimuli (composed of characters from the normal Apple text mode), declares those responses that will be accepted from the subject (who responds by typing at the Apple or a terminal keyboard), determines the absence/presence of and the type of response feedback, specifies pause durations, and establishes a randomization strategy for presenting stimuli. As in M.T.S., the sequence of several such tests may be determined from a master file that can be changed using the Pascal editor. Alternatively, the experiment may be carried out individually.
Often used procedures that deal with timing, screen presentation, etc., reside in a unit in the SYSTEM.LIBRARY.

Poltrock and Foltz (1982a) noted that their system, as described in 1982, allows little opportunity for response-contingent actions other than simple feedback or repetition of an item. Of course, the system also has limitations on size of test, number of items in a test, lengths of valid responses, and precision of event timing. They appropriately offered counsel regarding these matters in a manual (Poltrock \& Foltz, 1982b).

The Cognitive Testing Station (CTS) of Digitry, Inc., requires the same basic hardware as the Apple Pascal Tester. The CTS includes a printed circuit card that plugs into one of the Apple backplane slots and a pair of specially wired integrated circuit (IC) chips to replace two on the Apple motherboard. The printed circuit card contains a clock, logic to control the video output via the specially wired ICs, and a large number of parallel input/output lines to which multibutton response manipulanda and/or relays that may be used to control equipment are connected. As with the two other systems under discussion, the Digitry system provides its own library unit of procedures and functions to control timing, screen events, and input/output through the lines provided on the card. The Digitry system does not attempt to provide ready-made experimental procedures, but through a series of example programs shows how to access the capabilities of the system.

All three systems provide control of a timing device and a means to more completely control the video output. Of the three systems, the Apple Pascal Tester provides a means by which those with the least amount of detailed knowledge of the Pascal system can develop their own computer-controlled tests. The tests that may be developed, however, are somewhat limited as to input medium (keyboard only) and stimulus presentation (text screen) unless one has the ability to alter the Pascal source code of the system. The Cognitive Testing Station provides hardware/software support for easy access to a large number of input switches and output lines to control laboratory devices. It also provides ready-made hardware modifications that, when the other two systems are used, must be performed by the user. It would be expected that a commercial system such as Digitry's would also have the resources to more easily provide advice and support for its product than the other two systems, which are essentially free. The M.T.S. system provides many graphics-related features, including use of the $128 \mathrm{~K}$ RAM card as a graphics storage device and procedures to interface the touchscreen as a response device. The M.T.S. system also provides several tasks that have been developed and tested and a more integrated environment at the program level into which user-developed tasks may be included. Since all three systems employ the Apple Pascal system, it is fortunately possible to obtain the 
benefits of all three systems by combining those features from each that suit one's purpose.

\section{AVAILABILITY}

The M.T.S. system was made available for distribution on January 1, 1984. The library of low-level procedures, compiled code, and source code of the main program and individual tasks and full documentation of all aspects of the system, including detailed description of the hardware modifications, are available. For noncommercial use, there will be no charge except to cover costs of copying, handling, and mailing. Arrangements for commercial use may be made. In addition, consultation concerning the system and/or the implementation of new tasks may be arranged. An additional graphics development package that includes a full-color graphics editor and use of the 128K RAM card as a storage area for graphics is available for a fee from the first author. For a list of the available documents and programs, please contact the first author. The M.T.S. software is copyrighted by the first author.

\section{REFERENCES}

Poltrock, S. E., \& Foltz, G. S. (1982a). An experimental psychology laboratory system for the Apple 11 microcomputer. Behavior Research Methods \& Instrumentation, 14, 103-108.

Poltnock, S. E. \& Foltz, G. S. (1982b). User manual for Apple Pascal Tester.

Reed, A. V. (1979). Microcomputer display timing: Problems and solutions. Behavior Research Methods \& Instrumentation, 11, 572-576.

Appendix A

Contents of M.T.S. Intrinsic Units

The INTRINSIC UNIT MTSSTUFF consists of 13 lowlevel procedures and functions:

PROCEDURE TIMEPEEK reads 4 bytes of time data from the Mountain Computer Company Apple Clock and stores the data in a location passed to it as a parameter. The procedure reads the time and returns control to Pascal in about $0.65 \mathrm{msec}$. The data are not reformatted.

PROCEDURE TIMEFORM formats the time read by TIMEPEEK into seconds and milliseconds. This procedure requires about 14 msec to complete its task.

FUNCTION TIMEBETWEEN returns the time between the two unformatted times sent to it as parameters. The times sent are in the form returned by the procedure TIMEPEEK.

PROCEDURE PAUSE pauses for the time passed to it as a parameter. This procedure is not meant to be highly accurate; the actual pause is from 14 to $28 \mathrm{msec}$ longer than the time passed as a parameter.

FUNCTION HOMERESPONSE checks the foot switch and returns a Boolean value of TRUE if the switch is being pressed.

FUNCTION LEFTRESPONSE checks the left button and returns a Boolean value of TRUE if the button is being pressed.

FUNCTION RIGHTRESPONSE checks the right button and returns a Boolean value of TRUE if the button is being pressed.

PROCEDURE TIMEARESPONSE checks a specified combination of buttons (of the three available) and returns the time elapsed until one of the specified buttons was pressed and which button was pressed.

PROCEDURE AUDSTIM produces a tone of specified duration and frequency. Optionally, a response on one of the buttons will terminate the tone. It returns values that indicate the time from the start of the procedure until one button was released and the time until a second one was pressed.

PROCEDURE POKE, like the BASIC procedure of the same name, places a specified byte value into a specified location in memory.

FUNCTION PEEK, like the BASIC procedure of the same name, reads the value at a specified memory address.

PROCEDURE NOTHING does nothing. It is useful in that it makes null loops in Pascal easier to read in the source code.

The INTRINSIC UNIT MTSGRAFIX contains nine procedures that control HIRES graphics. Some of the functions performed by this unit duplicate similar procedures in the TURTLEGRAPHICS UNIT provided with Apple Pascal. MTSGRAFIX is completely compatible with TURTLEGRAPHICS but may be used without invoking TURTLEGRAPHICS. In its initialization, the MTSGRAFIX unit performs the same duties of loading the SYSTEM.CHARSET and protecting the HIRES screen.

PROCEDURE HIRESI switches the video output to the HIRES screen 1.

PROCEDURE TEXT1 switches the video output to the normal 40-column text screen 1.

PROCEDURE BLANKSCREEN interrupts all output to the video jack, thus blanking the video screen. It assumes the hardware modifications described in M.T.S. Technote 1.

PROCEDURE ONSCREEN restores the video output that was blanked via the BLANKSCREEN procedure. It assumes the hardware modifications described in M.T.S. Technote 1.

PROCEDURE SYNCHOFF turns off all video output just as the BLANKSCREEN procedure above, but does so in synch with the vertical synch signal. This procedure is especially useful in timing of video events and in providing smooth animation. It assumes the hardware modifications described in M.T.S. Technote 1.

PROCEDURE SYNCHON turns on the video just as procedure ONSCREEN above, but it does so in synch with the vertical synch signal. This procedure is very useful in specifying accurately when a visual stimulus is presented. Since the video screen refreshes only 60 times each second, it is important to know exactly when a particular sweep of the video screen begins. SYNCHON assumes the hardware modifications described in M.T.S. Technote 1.

PROCEDURE NEWSCREEN clears the HIRES screen to a specified background color (from the set black1, black2, white1, and white2) and initializes two variables that are used with the WRITESCREEN procedure below.

PROCEDURE WRITESCREEN is passed a string and an $x$ and a $y$ coordinate as parameters. The procedure writes the string to the HIRES screen beginning at the location passed. The characters used to write the string are those contained in the file SYSTEM.CHARSET. A character set that is specially constructed for use with the WRITESCREEN procedure is provided with the M.T.S. system. With the special character set, strings may be written in large characters in the colors violet, green, red, blue, and white. Colors may be intermixed, thus allowing use of contrasting color for emphasis. In addition, the character set may be altered to provide custom graphics characters to allow special character graphics effects or custom visual stimuli.

PROCEDURE PUTCHAR places one character from a specified file (of the same form as the file SYSTEM.CHARSET) at any specified location on the screen in any of four specified modes. This high-speed procedure provides complete control over both location and mode of presentation of the character. Normally, the character set used would be SYSTEM.CHARSET, but any number of character sets could be used from within the same program simply by specifying the location of the character set to be used when invoking the PUTCHAR procedure.

The INTRINSIC UNIT TOUCHRAM contains five procedures, three of which control input from the touchscreen and 
the remaining two of which provide special graphics features that employ use of the $128 \mathrm{~K}$ RAM card as a storage area for HIRES pictures.

PROCEDURE TPINIT initializes the touchscreen input mechanism. FUNCTION TPSTATUS returns an indication of whether a touch to the touchscreen has occurred since the last initialization of the touchscreen via the TPINIT procedure. A returned value of TRUE indicates that a touch has occurred.

PROCEDURE READTP reads touch information from the touchscreen. This information is sent as two characters representing $\mathrm{X}$ and $\mathrm{y}$ coordinates.

PROCEDURE KWIKRAMPIC transfers a HIRES picture from the 128K RAM card to the HIRES screen. With the KWIKRAMPIC procedure, the picture must be placed on HIRES byte boundaries in the $x$ dimension of the screen (these occur at every 7 th pixel along the horizontal dimension) but may be placed at any of the 192 y positions. Pictures may be of any size from 1 byte to an entire screen and may be placed on the screen in any of six modes that interact in different ways with information that may already be on the screen. The modes include replacing what is on the screen in the given position with the picture in Apple HIRES type 1 colors (violet, green, and white), replacing what is on the screen with the picture in type 2 colors (red, blue, and white), adding the picture to what is already on the screen, or "exclusively ORing" the picture onto the screen at the specified position. (When exclusively ORing the picture onto the screen, a pixel that is turned on in the picture will turn on a pixel on the screen unless that position on the screen is already on, in which case the screen pixel is turned off.) The two remaining modes place the picture on the screen using the full color information of each byte within the picture; thus, all six colors may be employed within the same picture. The picture may either replace what is already on the screen or be exclusively ORed with what is on the screen.

PROCEDURE HIGHRAMPIC transfers a HIRES picture from the 128K RAM card to the HIRES screen. The HIGHRAMPIC procedure allows the picture to be placed at any $x, y$ coordinate pair. To achieve the flexibility of positioning, the HIGHRAMPIC procedure loses some speed and control of color. Four modes of picture placement that correspond to the first four modes in KWIKRAMPIC are provided.

Both the KWIKRAMPIC and the HIGHRAMPIC procedures require pictures to be stored on the RAM card in a special form. The M.T.S. system package provides picture files that are loaded automatically by the system for each of the tasks that make use of these special graphics features. The form to which pictures must comply is detailed in the documentation. Alternatively, a utility program that performs all of the necessary details of picture placement and that includes a full-color graphics editor is available from the first author.

\section{Appendix B \\ Tasks Implemented on the M.T.S. System}

(1) Buttonpress training: This procedure should be used to begin each subject's first session. It trains the use of the two push buttons and the foot switch. It includes a check of accuracy of buttonpressing and a section that encourages rapid responding. It may be viewed as a crude basic literacy test.

(2) Simple auditory reaction time: The subject presses his/her preferred button after onset of a square-wave tone generated by M.T.S. software. A practice and a test are presented.

(3) Choice auditory reaction time: The subject must press a particular button (left for low pitch, right for high pitch) after onset of a tone. A practice and a test set are presented.

(4) Stroop color naming: The subject is asked to press the button that is the same color (red or blue) as a stimulus that is presented on the screen. The first set of stimuli are colored boxes. The second set of stimuli are color names ("BLUE" and "RED") that always agree with the color in which the word is written. The third set of stimuli consists of the same two words, again presented in either the color red or blue, but the color in which the word is written on the screen is uncorrelated with the word written.

(5) Forward digit span using touch telephone: After a short training set consisting of 3-, 4-, and 5-digit numbers, the subject is presented with numbers from 5 to 13 digits in length. The numbers are presented for $0.5 \mathrm{sec}$ per digit. The number is then replaced on the screen by an image of a large touch-tone telephone. The subject reproduces the number last seen by "dialing" in the number on the phone. Blocks of six numbers starting with 5 -digit numbers and increasing by 1 digit for each block are presented until the subject fails to correctly reproduce at least $50 \%$ of the numbers for two successive blocks. (This task uses the touchscreen and the $128 \mathrm{~K}$ RAM.)

(6) Learning supraspan digit strings via touch telephone: This task is presented as a second part of Task 5. Subjects are presented with a 15-digit number for $8.5 \mathrm{sec}$. The subject must then reproduce the number by dialing it on the telephone that appears on the screen. Each of five different numbers is present for five trials in a row or until the subject correctly reproduces the number. (This task uses the 128K RAM and touchscreen.)

(7) Visual masking: A focus point and orienting tone indicate the start of a trial. The focus point is replaced by one of the eight possible combinations of Xs and Os in a three-letter group. This stimulus stays on the screen for a brief interval, and then the screen is blanked for a brief interval. A masking stimulus is then presented in the position that formerly was occupied by the stimulus pattern. The mask is designed to exactly cover any pixel that could have been used by any stimulus combination. The mask remains on for 1 sec. The stimulus screen is then replaced by a display of the eight possible stimulus patterns. The subject must indicate which pattern he/she saw by touching it on the screen. The duration that the stimulus pattern remains on the screen is adjusted on the basis of the accuracy of the previous response. Trials are presented until a threshold value of known precision is determined. Sets of trials are given under two conditions of blank duration between stimulus and mask. (This task uses the touchscreen and the 128K RAM.)

(8) Visual choice reaction time with distractors: The subject must press the left or right button, depending upon the direction that a target stimulus points. During Set 1 , the target stimulus is presented alone. During Sets 2 and 3, the target stimulus is presented with a distractor stimulus that is somewhat similar to the target in shape. In Set 2, the distractor always points in the same direction as the target stimulus. In Set 3 , the direction that the target and distractor point is uncorrelated. (This task uses the 128K RAM.)

(9) Switching attention: position/number/color. During four initial sets, the subject is trained to respond differentially to target stimuli, depending on which of two words is on the screen. The target stimuli are the numerals 1 and 2, which may appear on the right or the left of the screen. When the word on the screen is "SIDE," the subject must press the left button if the target stimulus is on the left and press the right button if the target stimulus is on the right. When the word on the screen is "NUMBER," then the subject must press the left button (labeled "1") if the numeral is a 1 and press the right button (labeled " 2 ") if the numeral is a 2 . The word on the screen changes every two to eight trials. A third option that is available within the program but that is presently unused is to also use the dimension "COLOR," either replacing one of the above dimensions or in addition to "SIDE" and "NUMBER." (This task uses the 128K RAM.)

(10) Müller-Lyer illusion: The subject is presented with a line on the screen. The line has arrowheads pointing in the same direction on each end of the line and an arrowhead pointing in the opposite direction somewhere on the line between the two ends. The subject moves the middle arrowhead by pressing the left or the right buttons until the subject is ready to indicate that the line is divided in half by the middle arrowhead. Control conditions interspersed in the test set use vertical bars instead of 
arrowheads at the ends and in the middle. (This task uses the 128K RAM.)

(11) Was it there?: The subject is presented with two, four. or six digits for $0.5 \mathrm{sec}$ per digit, and then a probe digit is presented. The subject must indicate if the probe digit was included in the most recently presented string of digits. (This is the Sternberg procedure.)

(12) Simon Sez: One quadrant of the screen is illuminated with a color unique to that quadrant, and a tone unique to that quadrant is sounded. The subject must touch the quadrant that was illuminated. After a correct response, the first quadrant is illuminated again and then another quadrant is illuminated. The subject must now respond by touching the two quadrants in order. The sequence is extended until the subject responds incorrectly on two tries. Three sets, each with a different sequence, are presented. (This task uses the $128 \mathrm{~K} \mathrm{RAM}$ and the touchscreen.)

(13) Which word came last?: A series of words is presented one at a time. From time to time, the sequence is interrupted by a probe trial. In the probe trial, the subject is asked to indicate which of two indicated words came later in the series. The number of words intervening between presentation of the two probe words is varied from 0 to 4 , and the number of words that have been presented since the second of the two probe words is varied from 0 to 18 .

(14) Cups and Bowls: The subject is shown a stimulus dish along with two comparison dishes. The subject must indicate the comparison dish that is most similar to the stimulus dish. The dishes vary in width, depth, shape, and volume. (This task uses the touchscreen and the $128 \mathrm{~K} \mathrm{RAM}$.)

(15) Continuous recognition of words (with homophone distractors): A scries of words is presented one at a time. From time to time, the sequence is interrupted by a probe trial. In the probe trial, the subject must indicate whether a probe word was or was not presented within the series. Probe words are words from the series of words actually presented, words that are homophones of words from the series, or words that are neither in the series nor are hormophones of series words.

(16) Keeping track of objects in a house: Each of six objects is said to be placed in one of six locations in or around a house. The subject is shown a sentence describing each placement. After all objects are placed, the subject is asked if object $X$ is in location Y. Following the answer, the object is moved to a new location. The number of trials between placement and testing of an object's placement varies from zero to six.

(17) Keeping track of items in categories: Each of four categories is assigned one of four items from that category. After all four categories have been assigned an item, the subject is asked if the item in category $X$ is item $Y$. After the subject's response, the item assigned to that category is changed. The number of trials between assignment of an item and testing of the category varies from 0 to 12 .

In addition, other tasks, including tone-pattern recognition, visual-pattern recognition, and mental rotation, are in various stages of development. 